\title{
New active formulations against $M$. tuberculosis: Bedaquiline encapsulation in lipid nanoparticles and chitosan nanocapsules
}

\author{
L. De Matteis ${ }^{\mathrm{a}, 1, *}$, D. Jary ${ }^{\mathrm{b}, \mathrm{c}, 1}$, A. Lucía ${ }^{\mathrm{d}, \mathrm{e}, \mathrm{f}, 1}$, S. García-Embid ${ }^{g}$, I. Serrano-Sevilla ${ }^{g}$, D. Pérez ${ }^{\mathrm{h}}$, \\ J.A. Ainsa ${ }^{\mathrm{d}, \mathrm{e}, \mathrm{f}}$, F.P. Navarro ${ }^{\mathrm{b}, \mathrm{c}}$, J. M. de la Fuente ${ }^{\mathrm{g}, *}$ \\ ${ }^{a}$ Instituto de Nanociencia de Aragón, Universidad de Zaragoza and CIBER-BBN, Zaragoza, Spain \\ ${ }^{\mathrm{b}}$ Univ. Grenoble Alpes, F-38000 Grenoble, France \\ ${ }^{c}$ CEA, LETI, MINATEC Campus, F-38054 Grenoble, France \\ d Departamento de Microbiología (Facultad de Medicina), and BIFI, Universidad de Zaragoza, Zaragoza, Spain \\ ${ }^{\mathrm{e}}$ Instituto de Investigación Sanitaria Aragón (IIS-Aragón), Zaragoza, Spain \\ ${ }^{\mathrm{f}}$ CIBER de Enfermedades Respiratorias (CIBERES), Instituto de Salud Carlos III, Madrid, Spain \\ ${ }^{\mathrm{g}}$ Instituto de Ciencias de Materiales de Aragón, Universidad de Zaragoza/CSIC and CIBER-BBN, Zaragoza, Spain

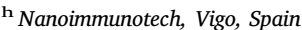

\section{G R A P H I C A L A B S T R A C T}

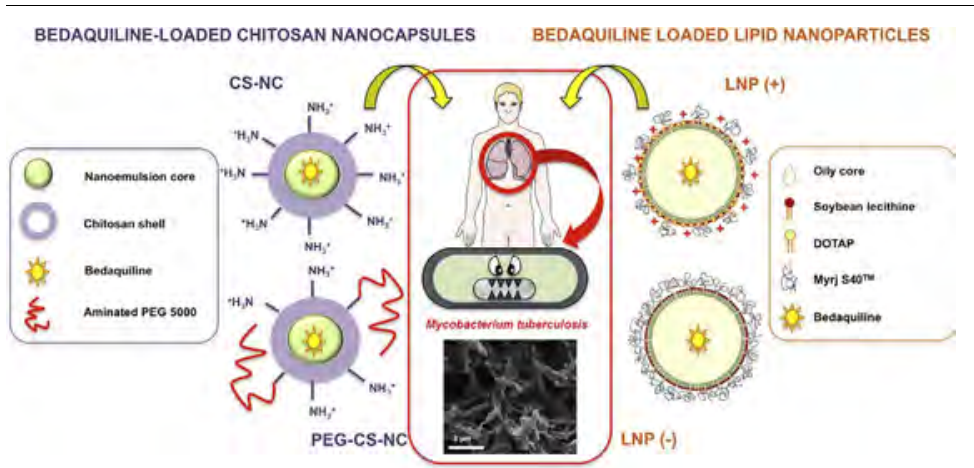

\section{A R T I C L E I N F O}

\section{Keywords:}

Nanocarriers

Bedaquiline

Mycobacterium tuberculosis

Electron microscopy

Cytotoxicicty

\begin{abstract}
A B S T R A C T
In the last years, the increase in antimicrobial resistance, together with a lack of new drugs for the treatment of bacterial infections resistant to classical antibiotics are of growing concern. Moreover, some of current therapies induce severe side effects and are often difficult to administer. In 2012 the FDA approved the use of bedaquiline, as the first new very effective drug against TB in the last 40 years. Despite its effectiveness, unfortunately bedaquiline side effects can be so dangerous that at present it is to be prescribed only when no other treatment options are available. The development of effective and safe nanotechnology-based methods can be particularly relevant to increase antimicrobial concentration at the site of infection, to reduce doses in the general circulation, which in turn reduces adverse effects. In this work bedaquiline was encapsulated in two types of nanocarriers, lipid nanoparticles and chitosan-based nanocapsules with high encapsulation efficiency and drug loading values. The efficacy of the drug-encapsulating nanocarriers has been demonstrated in vitro against Mycobacterium tuberculosis, together with the excellent compatibility of both carriers with animal cells. The obtained results open the way for further studies on multi-drug resistant strains of $M$. tuberculosis and for in vivo studies of the optimized nanocarriers. The promising behaviour of drug-loaded nanocarriers will hopefully lead
\end{abstract}

\footnotetext{
* Corresponding authors.

E-mail addresses: lauradem@unizar.es (L. De Matteis), jmfuente@unizar.es (J. M. de la Fuente).

${ }^{1}$ These authors contributed equally.
} 
to a reduction of the administered doses of a quite dangerous drug as bedaquiline, tuning its biodistribution and so decreasing its adverse effects, finally allowing its use in a higher number of patients.

\section{Introduction}

Novel drug delivery systems based on nanocarriers are a promising strategy to overcome current therapeutic limitations thanks to nanomaterials unique physicochemical properties. These include their small size, which allows them to reach the cellular level, their high surface to volume ratio, which increases interactions with target cells and their ability to be structurally and functionally modified to control their biodistribution. In addition, nanocarriers allow the improvement of aqueous solubility of poorly soluble drugs, the drug protection in order to avoid its degradation before reaching its target, its selective transport to the sites of infection and the controlled release of the medication to decrease the frequency of administration [1].

Many studies have been carried out for different anti-TB drugs, showing the success and usefulness of this technology to improve the treatment of tuberculosis [2].

The increase in antimicrobial resistance observed in last years is of growing concern worldwide and only thirteen new antibacterial agents were approved by the Food and Drug Administration (FDA) between 1999 and 2011 [3]. Mycobacterium tuberculosis (TB) have developed a high level of drug resistance to antimicrobial drugs. Multidrug resistant TB (MDR-TB) and extensively drug-resistant TB strains (XDR-TB, a form of MDR-TB with additional resistance to any of the fluoroquinolones and to at least one of three injectable second-line) have been identified in 105 countries worldwide, and they are making increasingly difficult to successfully treat tuberculosis. Drugs available for the treatment of bacterial infections resistant to antimicrobial drugs are very limited, induce severe side effects and/or are difficult to administer, so they could require parenteral injection [4]. Moreover the high costs of these treatments and their low success rates should be taken into account [5].

Bedaquiline, a diarylquinoline antimycobacterial drug, the first FDA approved anti-TB drug in four decades, is a very effective drug, nonetheless it shows serious side effects including induction of life-threatening cardiac arrhythmias [5]. Therefore, this drug is to be prescribed only when no other treatment options are available $[4,6]$.

The development of effective and safe nanotechnology-based methods would be particularly relevant to improve the safety of this drug for the treatment of drug-resistant infections. In fact, the use of a carrier can increase the local concentration of the drug at the site of infection, enabling lower doses in the general circulation, which in turn reduces the adverse effects.

Nanoemulsion-based nanocapsules present a lipidic core highly suitable for the encapsulation of a very hydrophobic molecule such as bedaquiline. For this reason, two bedaquiline-loaded nanocarriers have been developed in this work, consisting in a polymeric shell that surrounds a liquid or solid lipidic core where the drug is confined [7]. They are nanoemulsion-based chitosan nanocapsules (CS-NC), consisting in an oily core with a nanogel polysaccharidic shell grafted or not with polyethylene glycol molecules following a method previously described in literature by the same authors [8], and lipid nanoparticles (LNPs), made of a lipid core of long chains triglycerides and surrounded by a surfactant shell made of a polyethylene glycol based surfactant, phospholipids [9] and eventually cationic lipids in order to confer them a cationic character [10]. In both cases the polymeric coating has been used for nanocapsule stabilization [11].

In view of a possible finding of the optimal delivery system for bedaquiline, the two carriers have been designed for future, different administration route, being chitosan-based nanomaterials more suitable for nebulization and lipid nanoparticles very desirable for intravenous injection.
In the case of chitosan nanocapsules, bedaquiline has been encapsulated in the lipidic core and nanoemulsion was stabilized through chitosan coating by ionotropic gelation process. The same chitosanbased nanocapsules have been further grafted with a polyethylene glycol (PEG) layer to improve their colloidal stability in biologically interesting media. Among the high number of biopolymers used in drug delivery applications (alginate, hyaluronic acid, pectine, albumin, fibroin, etc.) [12,13], chitosan is widely used in nanomedicine because of the excellent biocompatibility and safety of its degradation products [14]. Chitosan has been chosen as nanoemulsion stabilizer since it is very rich in amino groups, which makes it useful to favour interaction of nanocarrier and respiratory tract mucose. Mucoadhesive properties of chitosan are especially important for aerosol administration for pulmonary infections $[15,16]$.

LNPs have been chosen as promising nanocarriers for this work because they gather several advantages for intravenous administration. They are composed of molecules generally regarded as safe, display no cellular toxicity and present long term circulation time due to their high colloidal stability and to the furtivity provided by the PEG shell $[17,18]$. LNPs with two different surface charges were studied, particles displaying a slightly negative surface charge (hereafter referred as LNPs $(-)$ ), due to the presence of zwitterionic lecithin molecules, and cationic ones $(\operatorname{LNPs}(+))$, containing DOTAP, a molecule with a quaternary ammonium polar head group. Both types of LNPs exhibited a PEG-rich outer shell, which is known to be a good strategy for drug delivery in the field of nanomedical therapy [19].

The encapsulation of bedaquiline has been optimized in the case of both carriers, upon quantification of drug loading and encapsulation efficiency. The resulting bedaquiline-loaded nanocapsules have been also characterized in terms of their physicochemical properties, since the knowledge of such characteristics is fundamental for the prediction and control of the interaction of nanomaterials with biological media and their toxicity [20]. Finally, chitosan nanocapsules and lipid nanoparticles have been compared in terms of their behaviour in biological systems. In particular, their in vitro antibacterial efficacy towards $M$. tuberculosis and their cytotoxicity in human cells have been established, prior to more in depth studies on multi-drug resistant TB strains and in vivo infected models.

\section{Materials and methods}

\subsection{Materials}

Tween $^{\circledast} 20$ and absolute EtOH, were purchased from Panreac Química S.L.U (Barcelona, Spain). Span ${ }^{\circledR} 85$ (sorbitanetrioleate), oleic acid and chitosan (medium molecular weight) were purchased from Sigma-Aldrich Pte. Ltd. (Singapore). Bedaquiline was obtained from AURUM Pharmatech LLC (Franklin Park, NJ, USA). Bis(sulfosuccinimidyl) suberate (BS3) was purchased from Pierce Biotechnology Inc. (Rockford, IL, USA) and $\alpha$-methoxy- $\omega$-amino poly(ethylene glycol) (PEG-MW 5000 Dalton) from IRIS Biotech GmbH (Marktredwitz, Germany). Myrj ${ }^{\mathrm{TM}}$ S40 (PEG 40 stearate, $1980 \mathrm{Da}$ ) and Super Refined Soybean Oil were obtained from Croda Uniquema (Chocques, France). Suppocire $\mathrm{NB}^{\mathrm{TM}}$ was purchased from Gattefosse S.A. (Saint-Priest, France). Lipoid ${ }^{\circledR}$ S75-3 (soybean lecithin at $69 \%$ of phosphatidylcholine) and hydrogenated S75 were provided by Lipoid GmbH (Ludwigshafen, Germany). All the products were pharmaceutical grade and used as received. DOTAP (1,2-dioleoyl-3-trimethylammoniumpropane (chloride salt)) was purchased from Avanti ${ }^{\circledast}$ Polar Lipids, Inc. (Alabaster, Alabama, USA). The fluorophore DiI (Molecular Probes ${ }^{\mathrm{TM}}$ Dil 
Stain, D282) was purchased from ThermoFisher Scientific (Illkirch, France).

Water (double processed tissue culture, endotoxin-free) used in all nanocapsule synthesis was from Sigma-Aldrich.

Plasma sample from healthy donor (Etablissement Français du Sang (EFS), Grenoble, FRANCE) collected by apheresis was used. According to the ethical and legal standards of our blood supplier (EFS), informed consent was given by blood donors. Blood usage was allowed by Health Department of Research Ministry as described in the delivered French directive \#DC-2008-334. The plasma pocket was delivered frozen and aliquoted in separated tubes kept at $-20^{\circ} \mathrm{C}$ for storage until use.

Bacterial strains used were Mycobacterium tuberculosis reference strain H37Rv [21] and Mycobacterium bovis BCG expressing the reporter Green Fluorescent Protein (GFP), both available at Mycobacterial Genetics Group Laboratory (University of Zaragoza). M. tuberculosis cultures were maintained in a BSL-3 laboratory.

Difco $^{\mathrm{TM}}$ Middlebrook 7H9 broth and BBLTM Middlebrook ADC Enrichment were purchased from BD (Becton, Dickinson and Company, Franklin Lakes, NJ, USA). Glycerol and Tween ${ }^{\circledR} 80$ were purchased from Scharlau. Resazurin sodium salt was from Sigma.

Cell lines THP-1 were and HepG2 were obtained from the ECACC: (cat. $\mathrm{N}^{\circ} 88,081,201$ and 85,011,430 respectively). Cell line A549 was available for this work at the University of Zaragoza.

Cell culture media, Roswell Park Memorial Institute medium (RPMI) and Dulbecco's Modified Eagle Medium (DMEM), used for cytotoxicity assays, cell culture maintenance and release profile assays, were obtained from Lonza (Basel, Switzerland). The Fetal Bovine Serum was from GIBCO. MTT (3-(4,5-dimethylthiazol-2-yl)-2,5diphenyltetrazoliumbromid) and Neutral Red were obtained from Sigma-Aldrich, as well as Phorbol 12-myristate 13-acetate (PMA).

Millipore Biomax $300 \mathrm{kDa}$ Ultrafiltration Discs and Millex syringedriven filter unit (PVDF and cellulose ones) with a pore size of $0.22 \mu \mathrm{m}$ and Isopore Membrane Filters, with a pore size of $0.1 \mu \mathrm{m}$ (VCTP), were purchased from Merck Millipore. Cellulose membranes used for lipid nanoparticles purification by dialysis at room temperature for $24 \mathrm{~h}$ in order to remove non-incorporated bedaquiline or any other ingredients (Regenerated cellulose, MWCO 12-14 kDa, ZelluTrans) were purchased from ROTH (Roth Sochiel E.U.R.L., Lauterbourg, France).

\subsection{Synthesis of bedaquiline-loaded nanocarriers}

The general procedure for the obtaining of nanoemulsion-based chitosan nanocapsules (CS-NC) has been previously reported in De Matteis et al. 2016 [8]. To encapsulate bedaquiline in nanocapsules, different amounts of drug were tested. 2, 3.5, 5, 10 or $25 \mathrm{mg}$ bedaquiline were mixed with $100 \mu \mathrm{L}$ absolute ethanol and $40 \mathrm{mg}$ oleic acid. This solution was then incorporated to an organic solution containing $8.6 \mathrm{mg} \operatorname{Span}^{\circledR} 85$ in $4 \mu \mathrm{L}$ absolute ethanol. The organic phase was then added drop by drop to an aqueous one containing $13.6 \mathrm{mg}$ Tween $^{\circledR} 20$ dissolved in $8 \mathrm{~mL}$ water under magnetic stirring during $15 \mathrm{~min}$ for the formation of the nanoemulsion core. In the next step, $0.5 \mathrm{~mL}$ of a $5 \mathrm{mg} / \mathrm{mL}$ chitosan solution in acetic acid $1 \%(\mathrm{v} / \mathrm{v})$ were added for the preliminary interaction of the polymer chains with nanoemulsion cores. Again, the mixture was left under stirring during $15 \mathrm{~min}$. Finally, the chitosan-coated nanoemulsion was added to $15 \mathrm{~mL}$ of $50 \mathrm{mM} \mathrm{Na}_{2} \mathrm{SO}_{4}$ under gentle stirring to obtain the final polymeric hydrogel shell. The excess of $\mathrm{Na}_{2} \mathrm{SO}_{4}$ was removed by ultracentrifugation $\left(69,673 \mathrm{G}, 30 \mathrm{~min}, 10^{\circ} \mathrm{C}\right)$, nanocapsules were washed with $10 \mathrm{~mL}$ of water, they were centrifugated again and resuspended in water. The concentration of the nanocapsules in water suspension was obtained by measuring the weight of a sample after freeze-drying.

For the grafting of chitosan nanocapsules with PEG5000, $20 \mathrm{mg}$ of nanocapsules at a concentration of $2 \mathrm{mg} / \mathrm{mL}$ in borate buffer $10 \mathrm{mM} \mathrm{pH}$ 8.3 were put in contact with an amount of $50 \mathrm{nmol} / \mathrm{mg}_{\mathrm{NC}}$ of bis(sulfosuccinimidyl) suberate $\left(\mathrm{BS}^{3}\right)$ and kept under stirring for $30 \mathrm{~min}$. A 1.5 excess of $\alpha$-methoxy- $\omega$-amino poly (ethylene glycol) (MeO-PEG- $\mathrm{NH}_{2}$ ) was added and, after $2 \mathrm{~h}$ of the reaction at $40^{\circ} \mathrm{C}, 20 \mathrm{~mL}$ of $10 \mathrm{mM}$ Tris$\mathrm{HCl}$ buffer $\mathrm{pH} 8.0$ were added to quench $\mathrm{BS}^{3}$ that eventually did not react with PEG. Grafted nanocapsules were filtered and washed with fresh water using an Amicon Ultrafiltration unit equipped with Millipore Biomax $300 \mathrm{kDa}$ Ultrafiltration Discs.

Lipid nanoparticles (LNP) were prepared by blending lipid and aqueous phases using ultrasonic process to provide stable nanodroplets. After homogenization at $55^{\circ} \mathrm{C}$, both phases are crudely mixed and sonication cycles are performed at $55^{\circ} \mathrm{C}$ during $5 \mathrm{~min}$. Non-encapsulated components are separated from Lipid Nanoparticles (LNPs) by dialysis with PBS, changing twice the equilibrating PBS buffer (with a volume equals $200 \times$ the LNP dispersion volume) and during $24 \mathrm{~h}$. Prior to characterization, LNPs dispersions are filtered through a $0.22 \mu \mathrm{m}$ cellulose membrane.

For LNPs(-), the lipophilic excipients, wax, oil and soya lecithin made up the oily phase whereas the hydrophilic PEG surfactant, MyrjS40 is solubilized in PBS as aqueous phase. By selecting the suitable weight ratios of core/shell excipients, $80 \mathrm{~nm}$-diameter of LNPs(-) were manufactured in $2.8 \mathrm{~mL}$-volume batches at $72 \mathrm{mg} / \mathrm{mL}$ of LNPs. The lecithin/PEG surfactant weight ratio was of 0.17 and the surfactant/core weight ratio is of 0.85 . For cationic LNPs, an additional cationic surfactant, DOTAP, was added as a lipophilic excipient. The lecithin/PEG surfactant weight ratio was of 0.051 and surfactant/core weight ratio was 0.67 . Two different weight ratios between oil and wax, $1 / 3$ and 3 , were tested for both LNPs.

Details about the weight percentage of the different components in anionic and cationic LNPs are reported in Fig. S3 in the Supplementary Information.

In both cases, the drug was incorporated in the lipid phase by mixing appropriate amount of bedaquiline stock solution $(10 \mathrm{mg} / \mathrm{mL}$ in dichloromethane), the solvent being completely evaporated at $55^{\circ} \mathrm{C}$ under Argon flow.

\subsection{Determination of bedaquiline encapsulation efficiency and drug loading}

Encapsulation efficiency (EE) was intended here as the percentage of encapsulated drug over the amount added initially to the preparation of nanocarriers. Drug loading (DL) is intended as the percentage of encapsulated drug per weight of carrier.

In the case of CS-NCs, to calculate the amount of encapsulated bedaquiline, $1.5 \mathrm{mg}$ of bedaquiline-loaded nanocapsules were mixed with $900 \mu \mathrm{L}$ methanol and sonicated for $30 \mathrm{~min}$, in order to obtain the complete extraction of the encapsulated drug. This solution was then filtered using a nylon syringe filter $(0.2 \mu \mathrm{m}$ pores $)$ to eliminate the chitosan fragments eventually present and the absorbance at $333 \mathrm{~nm}$ was measured using a Varian Cary $50 \mathrm{UV} /$ Vis spectrophotometer after carrying out a calibration curve of the compound in methanol. The encapsulated drug amount was also measured after extraction with dichloromethane from freeze-dried chitosan nanocapsules analysed with spectrophotometric analysis as previously described using a calibration curve in the same solvent.

Extracted bedaquiline was analysed also by HPLC method in a Waters 2695 HPLC system equipped with a Waters 2995 PDA detector and a HILIC column (HALO HILIC $4.6 \times 100 \mathrm{~mm}, 2.7 \mu \mathrm{m}$ ). Samples were injected $(10 \mu \mathrm{L})$ and eluted at $30^{\circ} \mathrm{C}$ and $0.5 \mathrm{~mL} / \mathrm{min}$ with an isocratic mixture of acetonitrile:water containing $0.2 \%$ formic acid (60:40). Sample quantification was carried out measuring the absorbance at $240 \mathrm{~nm}$, by means of a seven-point absolute calibration ranging from 1 to $110 \mathrm{ppm}$. The method was linear all over the measured range $\left(r^{2}=0.9991\right)$.

For LNPs, EE is first determined after final dialysis step of the preparation process. An additional method was necessary to be able to establish drug release profiles in different media as no simple method like centrifugation or filtration is available to separate LNPs from supernatant. To this purpose, two additional methods have been used for EE determination and the most appropriate one was chosen for drug 
release profiles. To determine the EE of bedaquiline in LNPs, the drug concentration in the purified dispersion, after intense dialysis, was measured by destabilization of LNPs in hydro-organic medium leading to liquid extraction of bedaquiline in solvent. Bedaquiline-loaded LNPs were diluted to 1:4 with acetonitrile, then vortexed to destroy the LNPs and release bedaquiline. A centrifugation of samples was then performed at $13,000 \mathrm{G}$ for $10 \mathrm{~min}$. The supernatant was gently withdrawn and transferred in convenient vial for analysis.

To be able to determine EE with a method alternative to dialysis purification, an aliquot of the dispersion was purified by SEC or by SPE [22]. The purified LNPs are then destabilized with the same method used for EE determination. For SEC purification, $200 \mu \mathrm{L}$ sample of LNP dispersion obtained after dialysis, at a concentration comprised between $15 \mathrm{mg} / \mathrm{mL}$ and $40 \mathrm{mg} / \mathrm{mL}$, was deposited gently on the top of Superdex 200 gel filtration media packed $(15 \mathrm{~cm}$ length) in a $15 \mathrm{~mm}$ inner diameter glass column (Kimble Chase Flex Column). Continuous flow of PBS at $0.7 \mathrm{~mL} / \mathrm{min}$ was applied and successive fractions of $500 \mu \mathrm{L}$ were collected in separate tubes. Optical absorbance at $315 \mathrm{~nm}$ of all fractions was measured in order to identify LNPs containing fractions. SPE was performed by using a SPE Waters Extraction manifold (20-position, $16 \times 75 \mathrm{~mm}$ tubes) connected to a vacuum pump (KnF, Germany). The SPE-cartridges Oasis ${ }^{\circledR}$ HLB $(6 \mathrm{~mL}, 150 \mathrm{mg}, 80 \AA$ pore size, $60 \mu \mathrm{m}$ particle size) composed of $\mathrm{N}$-vinylpyrrolidone and divinylbenzene polymers were also provided by Waters. In preliminary step, SPE-cartridges were conditioned by using $4 \mathrm{~mL}$ of acetonitrile and equilibrated with $4 \mathrm{~mL}$ of PBS. After loading $1 \mathrm{~mL}$ of nanoparticles sample, the non-adsorbed components were recovered after rinsing the column by adding $3 \mathrm{~mL}$ of PBS in a first fraction (F1), this fraction containing hydrophilic LNP. The free or shell-adsorbed bedaquiline was eluted in a second fraction (F2) by using $4 \mathrm{~mL}$ of acetonitrile. All steps were processed at 5 in Hg. Fractions F1 and F2 were both collected into $5 \mathrm{~mL}$-volumetric flasks and diluted to adjust volume to $5 \mathrm{~mL}$ with PBS and acetonitrile, respectively. Bedaquiline was then extracted in organic solvent from F1 fraction as described for EE determination.

Quantification of the drug has been performed by HPLC-UV with a HPLC Waters Alliance 2695 Separation module and a Waters 2487 UV detector equipped with a reversed phase SunFire C18. Elution was performed using a mobile phase composed of acetonitrile (ACN) and trifluoroacetic acid (TFA) in water at $0.1 \%$ with a linear gradient. The operating temperature was $25^{\circ} \mathrm{C}$ and the flow rate was $0.8 \mathrm{~mL} / \mathrm{min}$. Bedaquiline compound was eluted at a retention time of $7.8 \mathrm{~min}$ using a linear gradient mode from 50:50 TFA:ACN to 70:30 ACN:TFA with an injection volume of $20 \mu \mathrm{L}$ and the UV detection was recorded at $230 \mathrm{~nm}$. Standard curves with pure bedaquiline at 1, 2.5, 5, 7.5 and $10 \mu \mathrm{g} / \mathrm{mL}$ were performed for drug content determination in samples. The method was linear all over the measured range $\left(r^{2}=0.9999\right)$.

In the case of both carriers, to determine the long-term stability of drug loading, the previously described method was used to measure loading after six-month storage period in liquid suspension.

\subsection{Characterization of bedaquiline-loaded nanocarriers}

CS-NC: Dynamic Light Scattering (DLS) analysis has been carried out to obtain hydrodynamic diameter and polydispersity index (PDI) of nanocapsules using a Brookhaven 90Plus DLS instrument, by means of the Photo-Correlation Spectroscopy (PCS) technique. All measurements have been carried out in water at the concentration of $0.05 \mathrm{mg} / \mathrm{mL}$ at $25^{\circ} \mathrm{C}$.

Electrophoretic mobility ( $\zeta$ potential) of nanoparticles has been determined by measuring the surface potential of a $0.01 \mathrm{mg} / \mathrm{mL}$ nanoparticle suspension in $1 \mathrm{mM} \mathrm{KCl}$ at $25^{\circ} \mathrm{C}$ with a Plus Particle Size Analyzer (Brookhaven Instruments Corporation).

For the LNPs, the hydrodynamic diameter, polydispersity index and $\zeta$ potential of the lipid nanoparticles were measured with a Zeta Sizer Nano instrument (NanoZS, Malvern). The hydrodynamic diameter and PDI were measured with a $0.6 \mathrm{mg} / \mathrm{mL}$ LNP dispersion in PBS at $25^{\circ} \mathrm{C} . \zeta$
Potential was measured with a $0.4 \mathrm{mg} / \mathrm{mL}$ LNP dispersion in $1 \mathrm{mM}$ $\mathrm{NaCl}$, at $\mathrm{pH} 7.4$ and at $25^{\circ} \mathrm{C}$.

\subsection{Release study}

CS-NC bedaquiline-loaded nanocapsules were mixed with the proper medium at a concentration of $1.5 \mathrm{mg} / \mathrm{mL}$ nanocapsules and incubated at $37^{\circ} \mathrm{C}$. Samples were analysed after 1, 2, 3 and 7 days and bedaquiline retained was extracted from nanocapsules to be measured. Each solution was passed through a PVDF syringe filter $(0.2 \mu \mathrm{m}$ pores) to separate the nanocapsules (retained in the filter) from the medium. Then, $900 \mu \mathrm{L}$ of methanol were passed through the filter to extract bedaquiline still encapsulated. The concentration of bedaquiline in methanol was measured by spectrophotometric analysis at $333 \mathrm{~nm}$ and the amount of released drug was obtained by difference with the initial amount.

LNPs were prepared in each medium at a concentration of $6.7 \mathrm{mg}$ / $\mathrm{mL}$ of nanoparticles, corresponding to $200 \mu \mathrm{g} / \mathrm{mL}$ of bedaquiline and incubated at $37^{\circ} \mathrm{C}$, with a $350 \mathrm{rpm}$ stirring. After defined periods, an aliquot of the dispersion was collected for SPE purification and EE determination. The drug release percentage at time $t$ is defined by EE (t)-EE(t0).

\subsection{In vitro antimicrobial activity assessment}

Mycobacterium tuberculosis (reference strain, H37Rv) cultures were routinely grown at $37^{\circ} \mathrm{C}$ in Middlebrook $7 \mathrm{H} 9$ medium supplemented with $10 \%$ ADC and $0.05 \%$ Tween $^{\circledR}$ 80. Mycobacterium bovis BCG expressing GFP reporter was maintained in Middlebrook 7H9 medium supplemented with $10 \%$ ADC and $0.05 \%$ Tween $^{\circledast} 80$ with $50 \mu \mathrm{g} / \mathrm{mL}$ hygromycin.

The Minimum Inhibitory Concentration (MIC) was determined using the Resazurin Microtiter Assay Plate (REMA) [23]. In 96-well plates, a range of concentrations of the free bedaquiline or bedaquilineloaded nanoparticle were added in $100 \mu \mathrm{L}$ of Middlebrook 7H9 medium supplemented with $10 \%$ ADC and $0.5 \%$ glycerol in a series of twofold dilutions. Empty nanoparticles were assayed as a control. In this case, the range of final concentrations for bedaquiline spanned from 1 to $0.008 \mu \mathrm{g} / \mathrm{mL}$. Bacteria were inoculated by adding $100 \mu \mathrm{L}$ of a suspension of $10^{5} \mathrm{cfu} / \mathrm{mL}$, as estimated by optical density and prepared from a culture in exponential growth phase. After 6 days of incubation at $37^{\circ} \mathrm{C}$, $30 \mu \mathrm{L}$ of resazurin $(0.01 \% \mathrm{w} / \mathrm{v})$ was added to each well, incubated $48 \mathrm{~h}$ at $37^{\circ} \mathrm{C}$, and assessed for colour change. A change from blue to pink indicates bacterial growth. The MIC is defined as the lowest drug concentration that prevents this colour change.

\subsection{Scanning Electron Microscopy (SEM) analysis}

M. tuberculosis H37Rv cultures were grown for $48 \mathrm{~h}$ in the presence of the BQ-loaded nanocarriers, collected by centrifugation, washed once, resuspended in fixative solution $(2.5 \%$ glutaraldehyde in PBS $0.1 \mathrm{M}$ ) and kept for a minimum of $24 \mathrm{~h}$ at room temperature. The fixative solution was subsequently removed and the bacteria were washed three times with PBS $0.1 \mathrm{M}$ and filtered through a membrane with a pore size of $0.1 \mu \mathrm{m}$.

Filters with the bacteria and the nanocarriers were progressively dehydrated by immersion in a graded series of ethanol (50-100\%). SEM observations were performed using a field emission SEM Inspect F50 (FEI).

\subsection{Confocal Microscopy analysis}

M. bovis BCG expressing GFP was incubated at $37^{\circ} \mathrm{C}$ in the dark for $72 \mathrm{~h}$ with $\operatorname{LNPs}(-)$ labelled with the DiL fluorophore at a concentration of $200 \mu \mathrm{g} / \mathrm{mL}$. It was then washed once to remove the culture medium, and fixed with paraformaldehyde $4 \%$. The bacteria were washed twice 
with PBS to remove the fixative agent, and resuspended in water. One drop of the suspension was placed over the slide, air dried and mounted with moviol before visualization with the confocal microscope Zeiss LSM 880 Axio Observer, using an objective 63X/1.4 Oil DIC M27.

\subsection{Cytotoxicity assays}

Cell lines used in cytotoxicity studies were A549 (lung epithelial human cells), HepG2 (liver epithelial human cells) and THP1 (human monocytes from peripheral blood). A549 and HepG2 cells were cultured in T25 vented flasks with DMEM supplemented with $10 \%$ fetal bovine serum, penicillin and streptomycin, incubated at $37^{\circ} \mathrm{C} / 5 \% \mathrm{CO}_{2}$. For the assays they were seeded at a cell density of $10^{4} \mathrm{cell} / \mathrm{mL}$ for A549, and $2.5 \times 10^{4}$ cell $/ \mathrm{mL}$ for HepG2. THP-1 cells were cultured in T25 vented flasks with RPMI-1640 supplemented with $10 \%$ fetal bovine serum, penicillin and streptomycin. For the assays, cells were seeded at a density of $5 \times 10^{4} \mathrm{cell} / \mathrm{mL}$, and they differentiated into adherent macrophage-like cells by incubating with PMA at $5 \mathrm{ng} / \mathrm{mL}$ for $48 \mathrm{~h}$ at $37^{\circ} \mathrm{C} / 5 \% \mathrm{CO}_{2}$ [24]. After this period, the medium was changed to RPMI without PMA for $24 \mathrm{~h}$ more before they were exposed to the nanocarriers [24].

For the MTT viability assay, cells were seeded into a 96-well plate and maintained in culture for $24 \mathrm{~h}$ at $37{ }^{\circ} \mathrm{C} / 5 \% \mathrm{CO}_{2}$ to form a semiconfluent monolayer. They were then exposed to the nanocarriers using for this purpose DMEM or RPMI medium without phenol red, to avoid interference with the results. All nanocarriers were tested at 8 different concentrations prepared as twofold serial dilutions. The range of final concentrations tested was from $1 \mathrm{mg} / \mathrm{mL}$ to $7.81 \mu \mathrm{g} / \mathrm{mL}$. After $24 \mathrm{~h}$ of exposure, the culture medium was removed and $50 \mu \mathrm{L}$ of $1 \mathrm{mg} / \mathrm{mL}$ MTT was added to each well and incubated for $2 \mathrm{~h}$ at $37^{\circ} \mathrm{C} / 5 \% \mathrm{CO}_{2}$. After this incubation, supernatant was removed and the formed formazan crystals were solubilized in isopropanol. The colour intensity of the dissolved formazan is determined by photometric measurements at $570 \mathrm{~nm}$ and the percentage of viable cells was obtained by correlation to the intensity of the untreated control (100\% viability).

For Neutral Red Uptake (NRU) assay, cells were seeded into a 96well plate and maintained during $24 \mathrm{~h}$ at $37^{\circ} \mathrm{C} / 5 \% \mathrm{CO}_{2}$ to form a semiconfluent monolayer. They were then exposed to the nanocarriers using for this purpose DMEM or RPMI without phenol red, to avoid interference with the results. All nanocarriers were tested at 8 different concentrations prepared as twofold serial dilutions. The range of final concentrations tested was from $1 \mathrm{mg} / \mathrm{mL}$ to $7.81 \mu \mathrm{g} / \mathrm{mL}$. After $24 \mathrm{~h}$ exposure, the culture medium was removed and $100 \mu \mathrm{L}$ of $0.005 \%$ neutral red solution were added to each well and further incubated at $37^{\circ} \mathrm{C} / 5 \% \mathrm{CO}_{2}$ for $3 \mathrm{~h}$. The plates were then washed with PBS and the dye was extracted with ethanol/acetic acid solution before reading fluorescence of each well $(530 / 645 \mathrm{~nm})$. The number of viable cells was obtained by correlation to the intensity of the untreated control $(100 \%$ viability) [25].

\section{Results and discussion}

\subsection{Nanocarriers synthesis}

Due to the lipophilic character of bedaquiline, the lipidic core of both LNPs, made of vegetable oil and wax, and of CS-NC, based on nonionic surfactant/oleic acid micelles, have been found to be highly suitable for the encapsulation of this drug.

In this study the synthesis and characterization of the two kinds of nanocarriers is reported, from both a physicochemical and biological

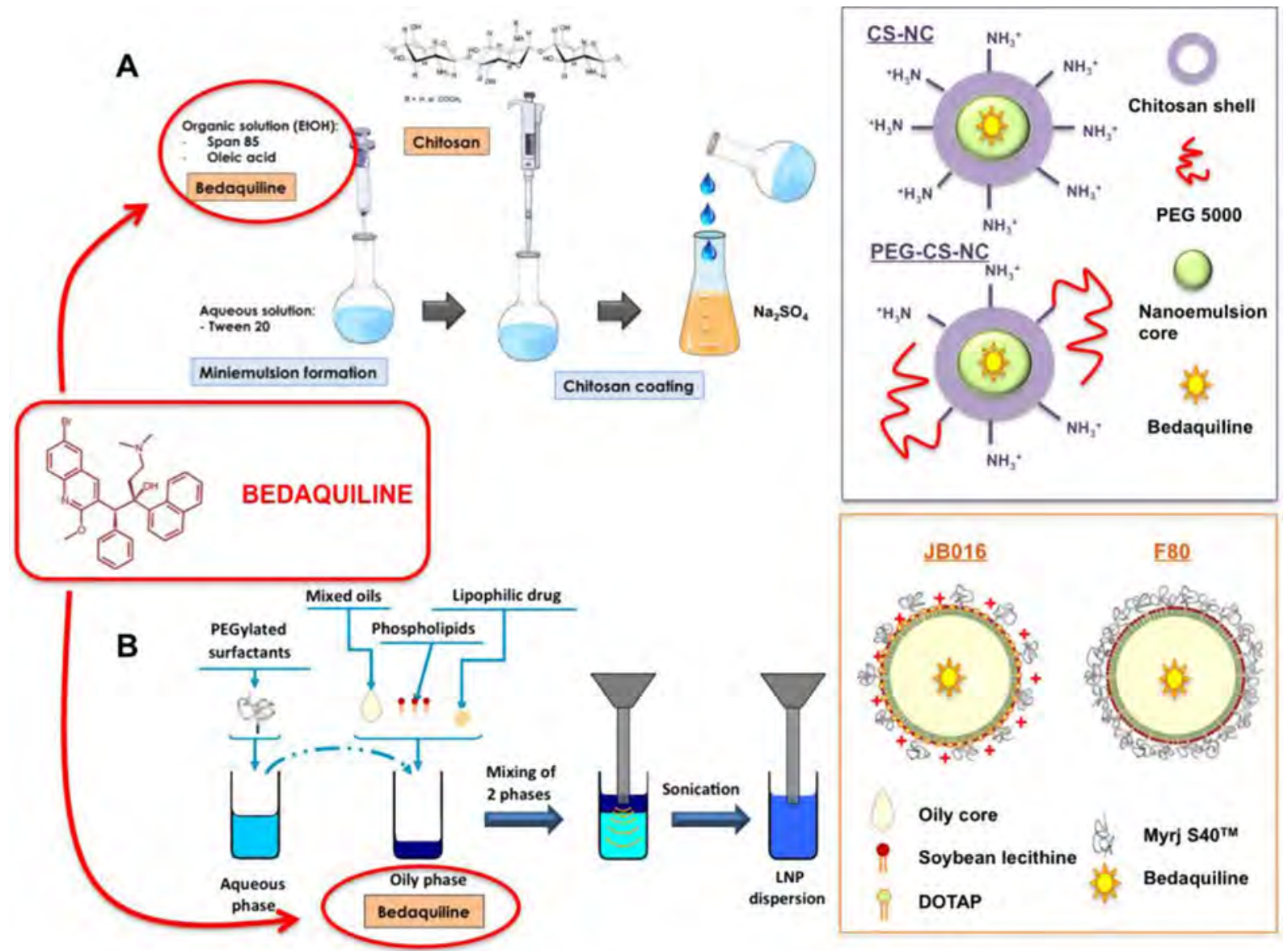

Scheme 1. Synthesis processes and schematic representation of the obtained nanocarriers used for bedaquiline encapsulation: CS-NCs (A) and LNPs (B). 
point of view, with the final aim of proposing them as candidates for future in vivo administration in the treatment of MDR-TB either by aerosol/nebulization or intravenous injection.

Since nanocapsules are intended as drug nanocontainers, the efficiency of drug encapsulation (EE, percentage of the encapsulated drug over the amount initially added) and the final drug loading (DL, percentage of the encapsulated drug per weight of obtained material) are important parameters to be optimized in view of biomedical application of the developed drug carriers. The optimization has been carried out with both kinds of nanocarriers. A description of the synthesis process and composition of bedaquiline-loaded nanocarriers is reported in Scheme 1.

\subsubsection{Chitosan nanocapsules}

In the case of CS-NCs the encapsulation of bedaquiline required a proper modification of the procedure already reported in literature [8] for the addition of bedaquiline to the organic phase of the synthesis mixture before obtaining the nanoemulsion suspension. The organic solution, containing the drug as well as the surfactant (Span $\left.{ }^{\circledR} 85\right)$ and the oil (oleic acid), is added to an aqueous solution of Tween ${ }^{\circledR} 20$ and nanoemulsion is spontaneously formed. At this step bedaquiline was included in the nanoemulsion core taking advantage of its hydrophobic nature. Finally the nanoemulsion was put in contact with the polysaccharide and this mixture was added to a sodium sulfate solution for the hydrogel formation. The whole process is schematically described in Scheme 1A. The grafting of the surface with aminated PEG5000 was carried out using $\mathrm{BS}^{3}$ as homobifunctional crosslinker and following a method already optimized in the case of empty nanocapsules [8]. Surface grafting offers the possibility to modify the behaviour of the nanocarrier in different media and depending on the desired function. Generally speaking, the modification of nanoparticles surface can be done to accomplish different objectives: i) to specifically recognize target tissue or cells, ii) to avoid the adsorption of serum proteins or the recognition by macrophages, and iii) to avoid aggregation in physiological medium $[19,26]$. The second and third objectives of the grafting are usually carried out by the attachment of hydrophilic molecules, such as polyethylene glycol, to coat the nanocarrier surface and eventually tune its properties. In this case, a surface grafting with PEG molecules of bedaquiline-loaded nanocapsules was performed to decrease their aggregation in culture medium and to study how such modification of their surface could affect cytotoxicity in human cells and their in vitro antimicrobial efficacy against $M$. tuberculosis.

To avoid eventual inter-particle crosslinking mediated by $\mathrm{BS}^{3}$ between amino groups on the surfaces, and so aggregation effects of the colloidal suspension of chitosan nanocapsules, the minimum amount of $\mathrm{BS}^{3}$ crosslinker was used to graft nanocapsule surface. $50 \mathrm{nmol} \mathrm{BS}{ }^{3} / \mathrm{mg}$ was found to be the optimal amount of crosslinker to allow the linking of PEG without any aggregation of the nanocarrier. After synthesis and grafting of nanocapsules, the encapsulated bedaquiline has been quantified using a method based on the extraction of the drug in organic solvent. The extraction was performed starting by both liquid suspension and freeze-dried nanocapsules and comparing results. Methanol and dichloromethane have been used respectively for the extraction of the drug from water suspension and dried material. The extracted compound could be easily measured by UV-Vis spectrophotometry after filtration of capsule fragments and interpolating results in a calibration curve previously obtained for each solvent. As the spectrophotometric analysis of bedaquiline in the extraction medium could suffer eventually from some interference of nanocapsules components, parallel measurements have been carried out with HPLC separation to further validate results obtained with spectrophotometric method. Drug loading has been optimized increasing the amount of bedaquiline initially added to the synthesis. Data referring to DL and EE of the optimization curve are reported in Fig. S1 of the Supplementary Information.

\subsubsection{Lipid nanoparticles}

Both anionic and cationic nanoparticles were optimized in terms of formulation, i.e. weight ratios of core excipients, and drug added during the preparation (data reported in Table S2 in Supplementary Information). The aim was to find the most stable LNPs in storage conditions with the highest EE. Lipid nanoparticles were prepared by blending lipid bedaquiline-containing phase and aqueous phase using ultrasonic process. Non-encapsulated components are separated from LNPs by dialysis with PBS. A first screening for material optimization has been made using the LNPs $(-)$ in order to optimize the formulation leading to the highest EE with a good stability. This optimization has then been translated to $\operatorname{LNPs}(+)$. The loading capacity of LNPs(-) with a core made of $25 \%$ of vegetable oil and $75 \%$ of wax (composition already used in previous studies [27]), has been studied. Different initial amounts of bedaquiline have been tested: $1 \%, 3 \%, 6 \%, 9 \%$ and $12 \%$ $(\mathrm{w} / \mathrm{w})$ showing that high LE, $6 \%$ and higher, lead to very rapid destabilization (within the 2 first weeks after batch preparation).

In order to improve stability of the LNPs during storage, also the composition of the lipidic core has been optimized. Three different ratios of solid lipids in the core have been studied for a six-month period stability: $0 \%, 25 \%$ and $75 \%$. It clearly appeared that the stability of LNPs(-) (3\% DL) is improved during storage at $4{ }^{\circ} \mathrm{C}$ by decreasing the ratio of solid lipids in the core, and a good stability has been maintained during six months without any significant EE decrease. The same kind of behaviour has also been observed with $\operatorname{LNPs}(+)$, presenting a DL of $3 \%(w / w)$, with identical EE after six months of storage at $4{ }^{\circ} \mathrm{C}$ compared to day 1 after preparation (data reported in Supplementary Information, Table S2). However, the comparison of DL and EE showed that the ratio between oil and wax in the core as well as surface charge of the LNPs did not influence the DL and EE of bedaquiline. After this screening step, LNPs( - ) and LNPs( + ) with a DL of $3 \%(\mathrm{w} / \mathrm{w})$ and a core made of $75 \%$ of vegetable oil and $25 \%$ of wax have been chosen as the best candidates for a further study to establish relationship between biological activity and properties of the surface of the LNPs.

As it is intended to establish drug release profiles in this work, a method more suitable than dialysis for EE determination was necessary. To that end, EE measurements after SEC purification and SPE purification have been compared to EE directly determined after dialysis of the batch. It appeared clearly that EE determined directly after dialysis is higher than what is obtained after further SEC or SPE purification. It is also important to note that SEC and SPE gave very close values of EE (Table S1 in Supplementary Information). A possible interpretation is that the drug loaded on the shell of the LNPs might be desorbed on the

Table 1

Nanocarriers characterization after bedaquiline encapsulation.

\begin{tabular}{|c|c|c|c|c|c|c|}
\hline Nanocarrier & DL, \% & $\mathrm{EE}, \%$ & Hydrodynamic diameter, nm & PDI & Z-potential, mV & Six-month stability, DL (6 months)/DL (t0), $\%$ \\
\hline CS-NC & $28 \pm 2$ & $70 \pm 7$ & $328 \pm 35$ & $0.151 \pm 0.027$ & $+26 \pm 4$ & $90 \pm 10$ \\
\hline PEG-CS-NC & $25 \pm 2$ & NA & $455.6 \pm 26$ & $0.204 \pm 0.020$ & $-9 \pm 2$ & $100 \pm 5$ \\
\hline $\operatorname{LNP}(-)$ & $2.8 \pm 0.15$ & $93^{*} \pm 6$ & $86 \pm 1.3$ & $0.148 \pm 0.005$ & $-10 \pm 1.1$ & $97 \pm 5$ \\
\hline $\operatorname{LNP}(+)$ & $2.8 \pm 0.15$ & $93^{*} \pm 7$ & $83 \pm 3.1$ & $0.123 \pm 0.009$ & $+28 \pm 3$ & $98 \pm 4$ \\
\hline
\end{tabular}

* EE determined by destruction of LNP and drug extraction in organic solvent after dialysis. 
reverse phase column during SPE extraction or desorbed on the purification gel when LNP flow through the gel during SEC purification. SPE purification appeared to be the most suitable for drug release profiles determination as it is easy to handle, very reproducible and without any possible contamination between successive samples [22].

\subsection{Nanocarriers characterization}

Chitosan nanocapsules, with or without PEG outer layer, underwent to physicochemical characterization to determine their physical characteristics (mainly size and surface potential) and to predict their behaviour in biological media.

Bedaquiline-loaded chitosan nanocarriers were characterized in terms of hydrodynamic diameter and $\zeta$ potential. A summary of characterization data, referring to both chitosan and lipid nanocarriers, is reported in Table 1. Hydrodynamic diameters are reported in terms of mean diameter. Drug loading and encapsulation efficiency percentages data have been also summarized in the same table and data referring to the stability of drug loading over time is also reported (expressed as ratio between DL measured after a period of 6 months from encapsulation, with nanocarrier stored at $4{ }^{\circ} \mathrm{C}$, and initial DL).

Hydrodynamic diameter of chitosan nanocapsules resulted to be significantly bigger than that of LNPs, possibly indicating a different internal structure of the carrier or a thicker polymeric shell. The differences in surface characteristics (charge and composition) did not affect significantly the size distribution of both chitosan and lipid nanocapsules.

The diameter distribution of chitosan nanocapsules, with and
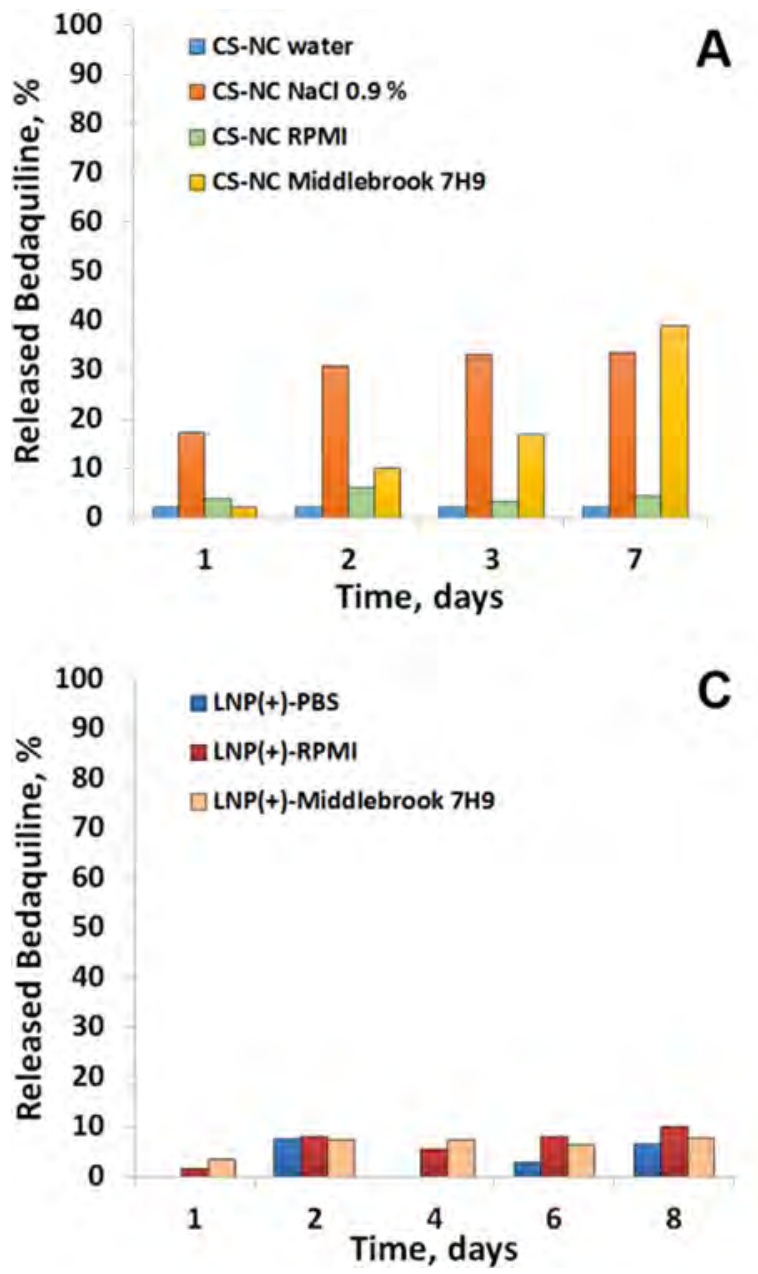

without PEG grafting, is also reported as count percentages corresponding to different diameters in Fig. S2 of Supplementary Information together with the same measurement in PBS to demonstrate the presence of PEG on grafted nanocapsules and its stabilizing effect. In fact, the aggregation effect observed for CS-NC in phosphate-based media was completely avoided after covalent linking of PEG on chitosan shell, demonstrating that the grafting was successfully achieved. Hydrodynamic diameter of chitosan nanocapsules was also measured in $\mathrm{NaCl} 0.9 \%$ to assess their stability. This medium has been taken into account because it will be interesting for future nebulization studies.

All nanocarriers (both chitosan nanocapsules and lipid nanoparticles) have been found to be slightly polydisperse as PDI values were all between 0.1 and 0.2 . Finally, data obtained from $\zeta$ potential measurements were in good agreement with the differences on nanocarrier surface in the case of both CS-NCs and LNPs, indicating the presence of a strong positive charge on nanocarrier surface in the case of LNPs(+) LNPs and not-grafted CS-NCs.

\subsection{Bedaquiline release profiles}

The profile of the release of bedaquiline from nanocarriers at $37^{\circ} \mathrm{C}$ was determined at fixed times and media selected on the basis of conditions used in the in vitro assays. Middlebrook 7H9 is the medium used in M. tuberculosis cultures and RPMI medium was also taken into account since it is used for infected macrophages cultures, so the behaviour of nanocarrier in this medium could be of interest for future in vitro studies. Release was also measured in the storage media (water and PBS for CS-NC and LNP respectively) and $\mathrm{NaCl} 0.9 \%$ was used in
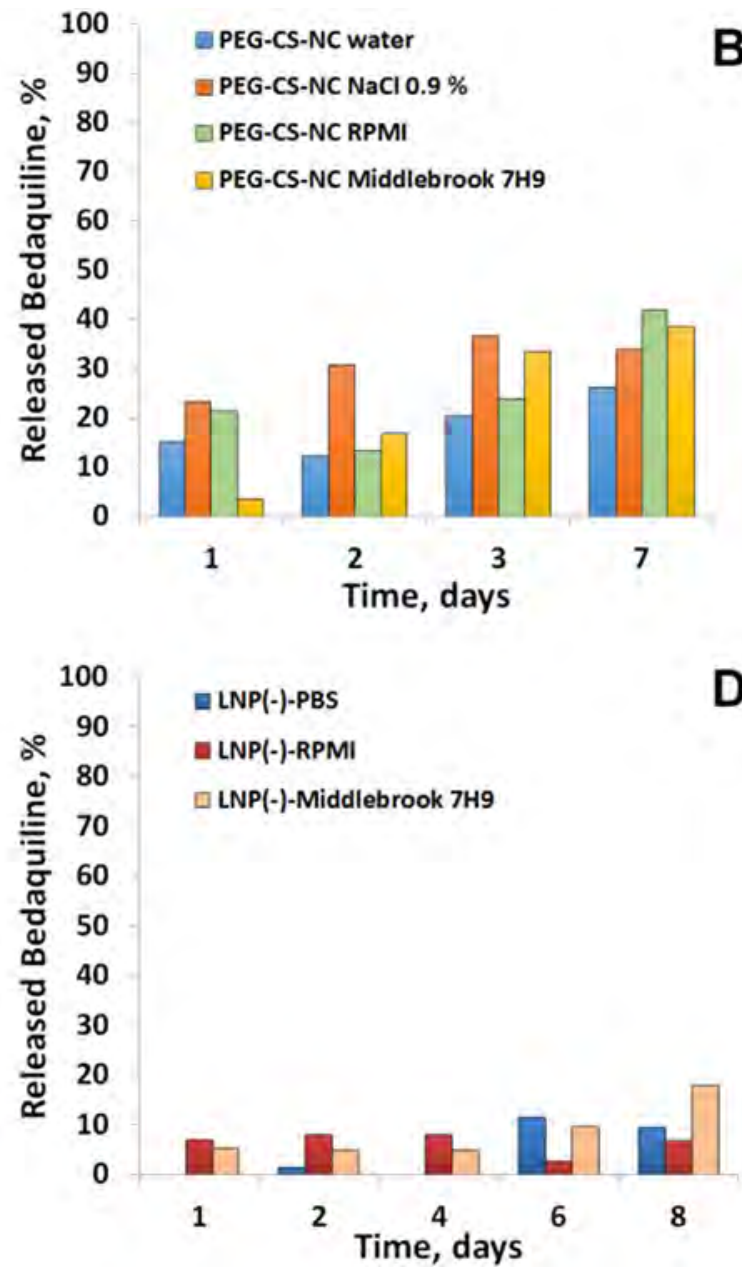

Fig. 1. Release profiles of LNPs and CS-NC in different media of biological interest. CS-NC (A), PEG-CS-NC (B), LNPs (+) (C) and LNPs (-) (D). 
the case of CS-NCs in view of the suitability of this carrier for nebulization. Percentages of released bedaquiline at different incubation times are reported in Fig. 1.

LNPs loaded with bedaquiline were found to be very stable in PBS, RPMI and Middlebrook 7H9 medium (in all cases less than 10\% drug was released after one week). The release of bedaquiline from CS-NC in Middlebrook $7 \mathrm{H} 9$ broth and in $0.9 \% \mathrm{NaCl}$ was more significant, reaching almost $40 \%$ after 7 days of incubation. However, release was found to be quite slow but sustained all along the experiment, with $20 \%$ released after 3 days. On the contrary, $\mathrm{NaCl}$ seems to favour a slightly faster release. In fact, $40 \%$ of drug was released after $48 \mathrm{~h}$, although this value remained unchanged after 7 days. Chitosan nanocapsules grafted with PEG on their surface showed the same release profile in $\mathrm{NaCl}$ and Middlebrook 7H9 medium. Nevertheless, grafting seems to favour bedaquiline release in both water and RPMI medium, in which released percentages reached the same value than in other media after 7 days incubation. These differences were attributed to the changes in nanocapsule surface that would strongly affect the interaction of nanocapsules with proteins and salts contained in Middlebrook 7H9 and RPMI media. A slightly higher release has been observed in water using grafted nanocapsules. The difference with non-grafted nanocarriers could be addressed to the use of borate buffer during the grafting process. In fact, it could possibly promote some leakage of sulphate ions from the polymeric network, that could negatively affect its pore size and so slightly increase the diffusion.

Release in human plasma was also studied in the case of LNPs for future intravenous injection applications and data are reported in Fig. 2.

\subsection{In vitro antimicrobial activity and cytotoxicity studies}

The in vitro antimicrobial activity of the nanocarriers against $M$. tuberculosis H37Rv was determined using the well-established REMA assay [23], and compared to that of free bedaquiline (Table 2).

The MIC of empty carriers was determined as well, to ensure that, within the range of concentrations used in the assay, the antibacterial effect was only due to the antimicrobial drug and not to the nanocarrier itself (data not shown). As shown in Table 2, bedaquiline was still very active against $M$. tuberculosis after encapsulation in any of the carriers. These results were nicely reproduced a minimum of five times, using different batches of capsules.

As first approach, prior to more accurate, future in vivo toxicity studies, and as first screening of the effect on human cells of nanocarriers with encapsulated bedaquiline, cell viability was also determined in in vitro studies using different tests and different cell lines, to increase the reliability of the results obtained. The cytotoxicity of the four bedaquiline-loaded nanocarriers was assessed by two different methods, MTT and NRU, which are the most sensitive assays in detecting cytotoxic events [28].

MTT is the best-known method for determining cellular proliferation by measuring the conversion of MTT in the mitochondria due to dehydrogenase activity in the living cells. NRU determines the accumulation of the neutral red dye in the lysosomes of viable, uninjured cells. The method allows an accurate and reliable quantification of the viable cells after each treatment[25]. Neutral red uptake assay has been used for the estimation of cell viability [25]. Both methods were assayed in three different cell lines: lung epithelial human cells (A549) because M. tuberculosis infection is located in the lungs, liver epithelial human cells (HepG2) as the nanoparticles would circulate through the liver, and human monocytes from peripheral blood (THP-1), chosen because M. tuberculosis is an intracellular pathogen that resides inside the macrophages. Results are reported in Table 3.

For the LNPs, the cytotoxicity of the particles was detected only at very high concentrations, over $1 \mathrm{mg} / \mathrm{mL}$. For CS-NCs cytotoxic effect appears over $0.25 \mathrm{mg} / \mathrm{mL}$ nanocapsules (70\% of survival). However, for both carriers, tolerated concentrations are in all cases far superior to those needed to reach the minimum inhibitory concentration of the drug, i.e. $0.1-0.94 \mu \mathrm{g} / \mathrm{mL}$ and $1-2 \mu \mathrm{g} / \mathrm{mL}$ respectively in the case of CSNC and LNP. In other words, none of the bedaquiline-loaded nanocarriers developed have any cytotoxic effect on A549, HepG2 and THP1 cell lines at the concentration needed to kill the bacteria.

\subsection{Interaction between bacteria and nanoparticles}

As preliminary attempt to study the interaction responsible for the effect of nanocarriers on $M$. tuberculosis, a microscopic analysis has been carried out to evaluate a possible approach or interaction of the particles with the mycobacterial envelope. Scanning Electron Microscopy has been used in the case of chitosan nanocapsules and Confocal Microscopy in the case of lipid nanoparticles due to the sensitiveness of these carriers to SEM conditions. The aim of the study was to evaluate if the particles were able to locate next to the bacteria and release the drug closer to or even inside the bacteria. That would be an important aspect to take into account for a treatment, since encapsulation would help to reduce the dose of the drug needed. SEM images of $M$. tuberculosis incubated with chitosan nanoparticles are reported in Fig. 3B and C, together with a control of the mycobacteria that have not been incubated with nanocarriers (Fig. 3A).

The carriers were abundantly observed in strict contact with the bacteria, independently of the presence of PEG on their surface, apparently forming clusters of nanocapsules.

On the other hand, since LNPs do not stand the dehydration needed to prepare the samples for SEM, another approach was used to visualize them. LNPs $(-)$ were labelled with the fluorophore DiL $(520 / 570 \mathrm{~nm})$ and, after incubation with the bacteria, the samples were observed with the confocal microscope. To help in visualization, a strain of Mycobacterium bovis BCG (an antivirulent strain used worldwide currently for vaccination against tuberculosis) expressing GFP was used for this experiments instead of $M$. tuberculosis H37Rv. Images are reported in Fig. 4. Also in this case, the LNPs( - ) were found around the bacteria, but surrounding the bacterial cell in a more homogeneous distribution, indicating again that this close interaction of nanocarriers with the bacterial envelope could have possibly an important role in the antibacterial activity observed.

\section{Conclusions}

Nanocarriers developed in this work were able to encapsulate bedaquiline, a very effective, although dangerous anti-TB drug, with excellent efficiency and very good drug loading values and the work

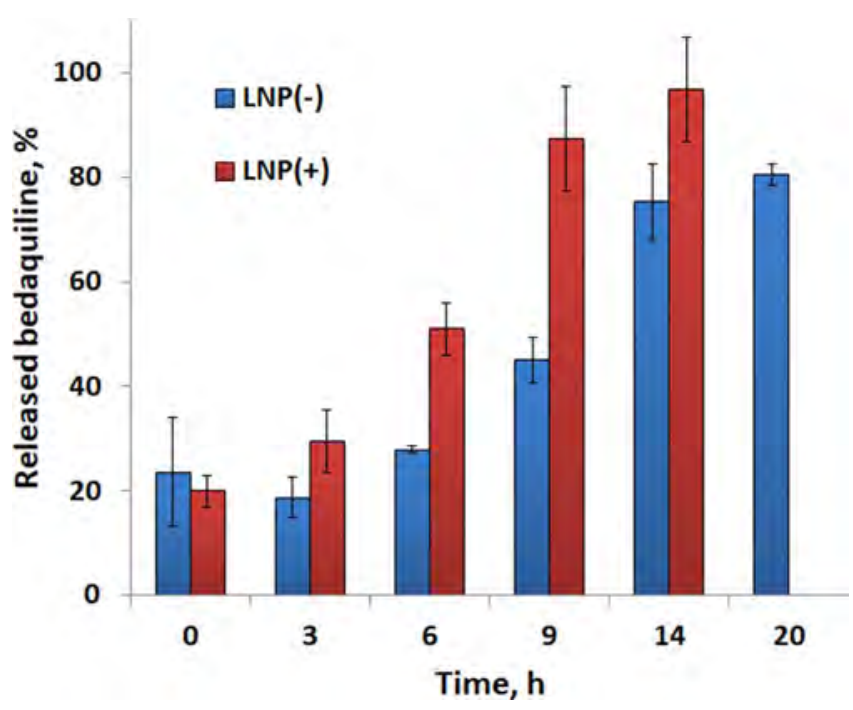

Fig. 2. Release profiles of LNPs in human plasma. 
Table 2

MIC values of bedaquiline-loaded nanocarrier.

\begin{tabular}{lll}
\hline Nanocarrier & $\begin{array}{l}\text { MIC } \\
(\mu \mathrm{g} / \mathrm{mL} \text { of BQ })\end{array}$ & $\begin{array}{l}\text { Corresponding concentration of nanocarrier } \\
(\mu \mathrm{g} / \mathrm{mL})\end{array}$ \\
\hline Free BQ & 0.03 & - \\
CS-NC & $0.03-0.06$ & $0.1-0.4^{*}$ \\
PEG-CS-NC & $0.06-0.13$ & $0.48-0.94^{*}$ \\
LNP( $(-)$ & 0.03 & $1-2$ \\
LNP(+) & 0.03 & $1-2$ \\
\hline
\end{tabular}

* Range of concentrations taking into account all the batches tested.

Table 3

MTT and NRU results in different cell lines.

\begin{tabular}{|c|c|c|c|c|c|c|}
\hline \multirow[t]{3}{*}{ Nanocarrier } & \multicolumn{6}{|c|}{$\begin{array}{l}\text { Nanocarrier concentration corresponding to } 70 \% \text { of viability }(\mu \mathrm{g} / \mathrm{mL} \text { of } \\
\text { nanocarrier) }\end{array}$} \\
\hline & \multicolumn{3}{|l|}{ MTT } & \multicolumn{3}{|l|}{ NRU } \\
\hline & A549 & HepG2 & THP1 & A549 & HepG2 & THP1 \\
\hline CS-NC & $100-150$ & 350 & $150-200$ & $200-250$ & 300 & 50 \\
\hline PEG-CS-NC & $50-100$ & 350 & 300 & $150-200$ & 100 & ND \\
\hline $\operatorname{LNP}(-)$ & 850 & $>1000$ & $>1000$ & $>1000$ & $>1000$ & $>1000$ \\
\hline $\mathrm{LNP}(+)$ & 600 & $>1000$ & $>1000$ & $>1000$ & 250 & $>1000$ \\
\hline
\end{tabular}

represents the first bedaquiline encapsulation, at the best of our knowledge. The aim of the work was to obtain the development and fundamental physicochemical characterization of this promising nanocarriers that would open the way in the future for more in depth studies to verify the activity of encapsulated bedaquiline against MDRTB and investigate the expected decrease in treatment toxicity.

Results obtained were very encouraging, in fact the in vitro antimicrobial activity of the drug against TB strain H37Rv was maintained almost unchanged after encapsulation in all the nanocarriers, indicating that the drug has not been damaged in the process of encapsulation, and that after encapsulation it is still as effective as the free drug. Moreover no cytotoxic effect on A549, HepG2 and THP-1 cell lines of bedaquiline- loaded nanocarriers was observed at the concentration needed to kill the bacteria.

From the obtained results, it can be concluded that both kinds of carriers represent very good candidates to be tested for in vivo activity and toxicity hopefully in view of a safer administration of this drug.

The absence of any burst release of the encapsulated drug in all the tested nanocarriers, let us hypothesize that the release of the drug could happen more probably once the material enters in contact with the bacterial cell. Preliminary microscopy experiments on the interaction of nanocarriers with bacterial cells indicated the close interaction of nanocarriers with the bacteria surface. The proximity of nanocarriers to bacterial envelope could indicate their possible entrance in the cell or a delivery of the drug once in contact with the cell wall, taking into account the lipidic nature of the core of both kind of materials and the presence of highly hydrophobic mycolic acids in the cell wall of mycobacteria.

In conclusion, this work provides an interesting starting point for the possible future improvement of bedaquiline-based treatments of $M$. tuberculosis infections and highlight the need to study more in depth the interaction of nanomaterials with microbial cell to allow us to obtain more and more efficient nanocarriers for drug delivery.

\section{Acknowledgements}

Authors would like to acknowledge the public funding from Fondo Social de la DGA (grupos DGA), Ministerio de la Economía y Competitividad del Gobierno de España for the public founding of ProyectosI + D + i - ProgramaEstatal de Investigación, Desarrollo e InnovaciónOrientada a los Retos de la Sociedad (project n. SAF201454763-C2-2-R), the European Seventh Framework Program (NAREB Project 604237) and Ministerio de Educación Cultura y Deporte for SGE fellowship. We also want to acknowledge Dr. C. Cuestas Ayllon for his technical support in SEM experiments and the Advanced Microscopy Laboratory (LMA) of Universidad de Zaragoza as well as César Vallejo and María Royo from Confocal Microscopy facilities at Universidad de Zaragoza. We would like also to acknowledge Dr. Estela Solanas and Dr. Nacho Aguiló for kindly provide us cell lines for cytotoxicity studies.
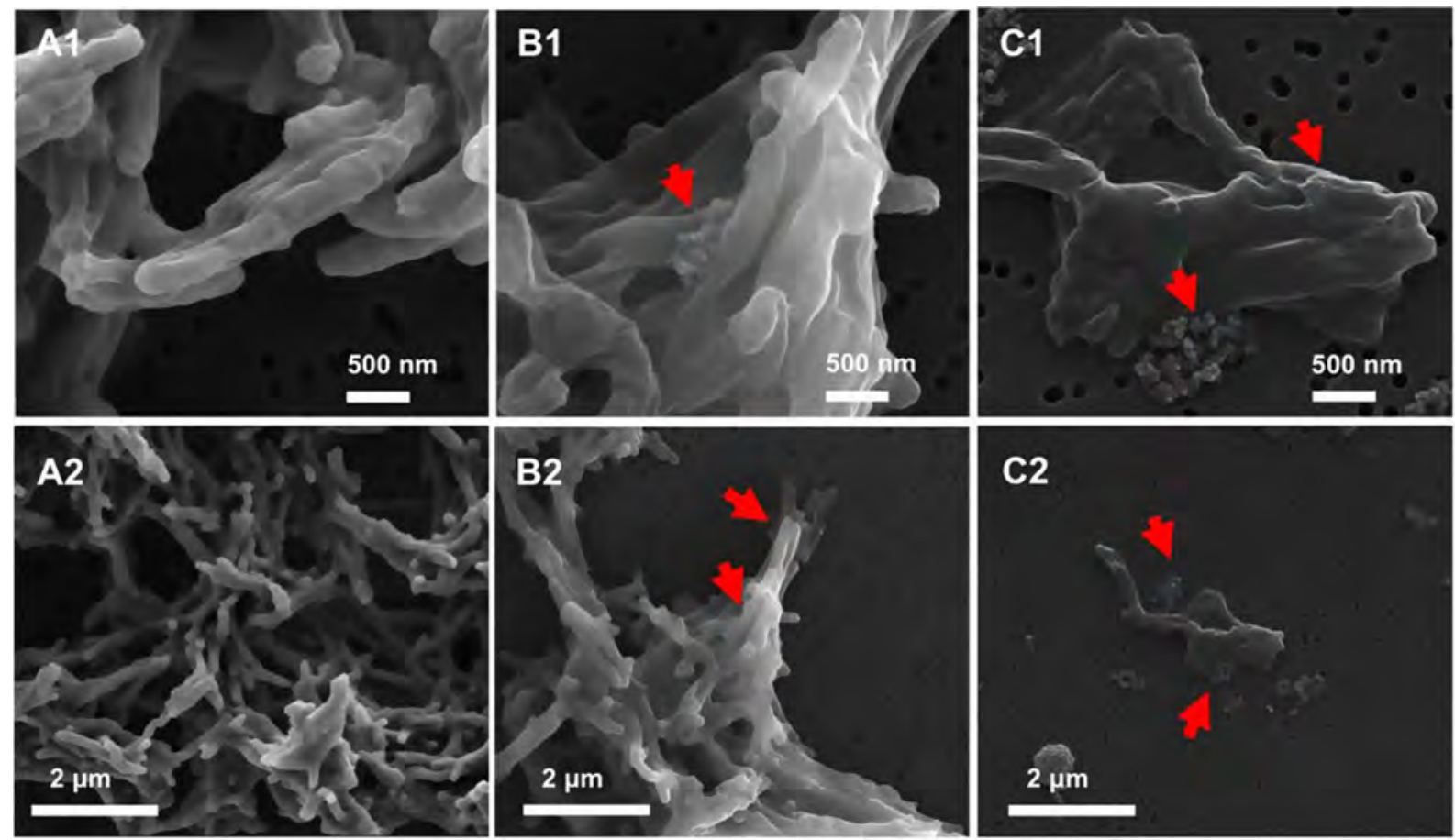

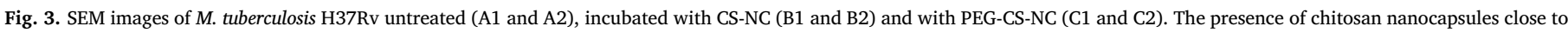
the bacterial cells is indicated by the arrows. 

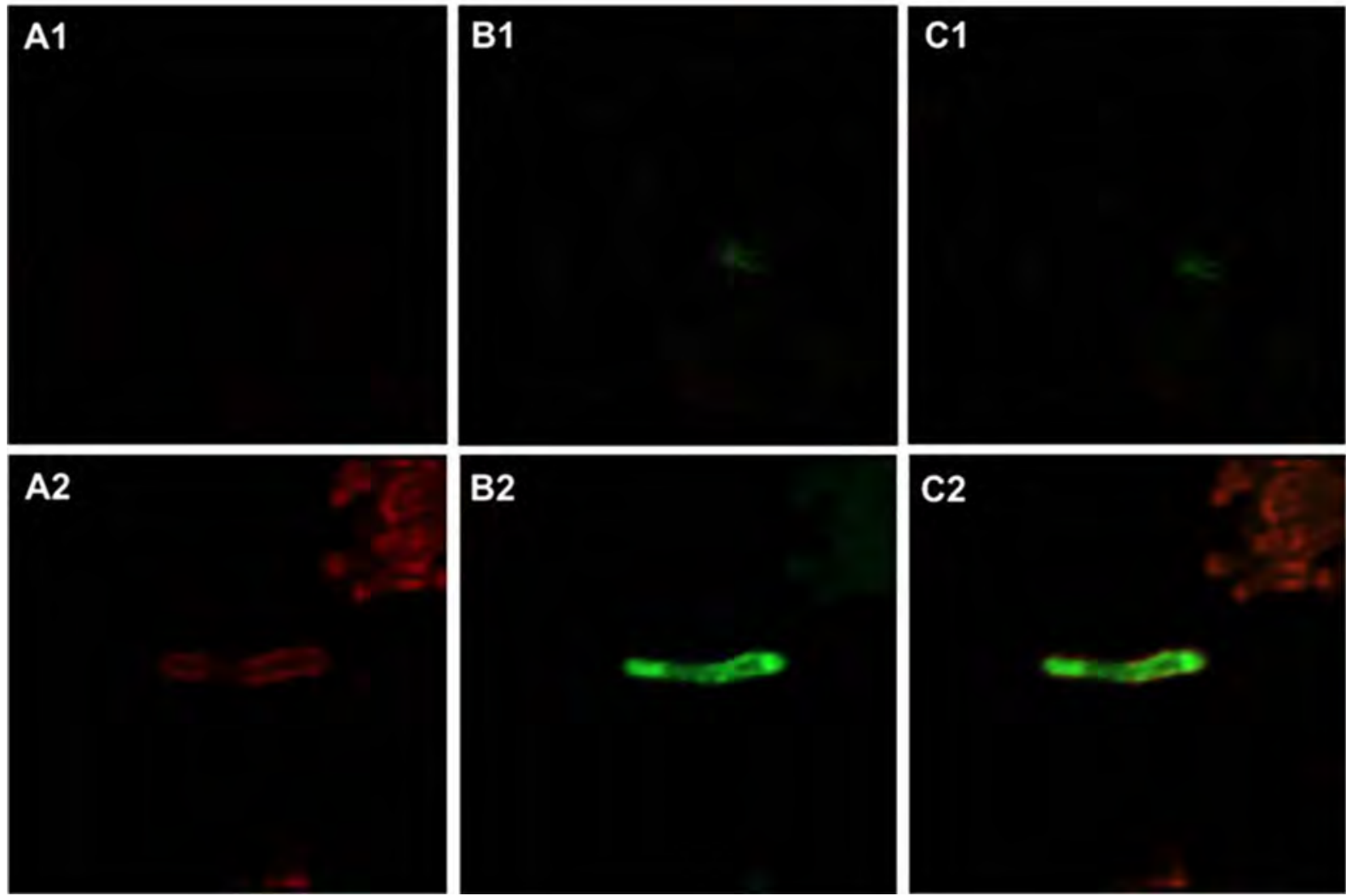

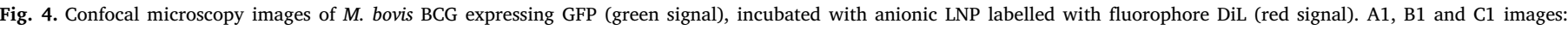

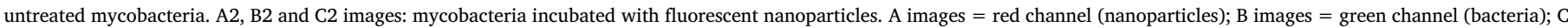

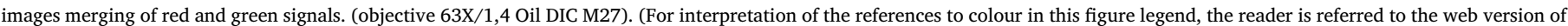
this article.)

Authors also acknowledge Servier Medical Art by Servier for graphical support.

\section{Appendix A. Supplementary data}

Supplementary data associated with this article can be found, in the online version, at http://dx.doi.org/10.1016/j.cej.2017.12.110.

\section{References}

[1] P. Boisseau, B. Loubaton, Nanomedicine, nanotechnology in medicine, Comptes Rendus Phys. 12 (2011) 620-636, http://dx.doi.org/10.1016/j.crhy.2011.06.001.

[2] J. Costa-Gouveia, J.A. Aínsa, P. Brodin, A. Lucía, How can nanoparticles contribute to antituberculosis therapy? Drug Discov. Today. 22 (2017) 600-607, http://dx.doi. org/10.1016/j.drudis.2017.01.011.

[3] R.S. Kalhapure, N. Suleman, C. Mocktar, N. Seedat, T. Govender, Nanoengineered drug delivery systems for enhancing antibiotic therapy, J. Pharm. Sci. 104 (2015) 872-905, http://dx.doi.org/10.1002/jps.24298.

[4] S. Mustafa, R.S. Pai, G. Singh, V. Kusum Devi, Nanocarrier-based interventions for the management of MDR/XDR-TB, J. Drug Target 23 (2015) 287-304, http://dx. doi.org/10.3109/1061186x.2015.1009076.

[5] C. Lange, I. Abubakar, J.W.C. Alffenaar, G. Bothamley, J.A. Caminero, A.C.C. Carvalho, K.C. Chang, L. Codecasa, A. Correia, V. Crudu, P. Davies, M. Dedicoat, F. Drobniewski, R. Duarte, C. Ehlers, C. Erkens, D. Goletti, G. Günther, E. Ibraim, B. Kampmann, L. Kuksa, W. De Lange, F. Van Leth, J. Van Lunzen, A. Matteelli, D. Menzies, I. Monedero, E. Richter, S. Rüsch-Gerdes, A. Sandgren, A. Scardigli, A. Skrahina, E. Tortoli, G. Volchenkov, D. Wagner, M.J. Van Der Werf, B. Williams, W.W. Yew, J.P. Zellweger, D.M. Cirillo, Management of patients with multidrugresistant/extensively drug-resistant tuberculosis in Europe: a TBNET consensus statement, Eur. Respir. J. 44 (2014) 23-63, http://dx.doi.org/10.1183/ 09031936.00188313.

[6] S.C. Goulooze, A.F. Cohen, R. Rissmann, Bedaquiline, Br. J. Clin. Pharmacol. 80 (2015) 182-184, http://dx.doi.org/10.1111/bcp.12613.

[7] A. Zimmer, J. Kreuter, Microspheres and nanoparticles used in ocular delivery systems, Adv. Drug Deliv. Rev. 16 (1995) 61-73, http://dx.doi.org/10.1016/0169409X(95)00017-2.

[8] L. De Matteis, M. Alleva, I. Serrano-Sevilla, S. García-Embid, G. Stepien, M. Moros, J.M. De La Fuente, Controlling properties and cytotoxicity of chitosan nanocapsules by chemical grafting, Mar. Drugs 14 (2016) 175, http://dx.doi.org/10.3390/ md14100175.

[9] T. Delmas, A.C. Couffin, P.A. Bayle, F. de Crécy, E. Neumann, F. Vinet, M. Bardet, J. Bibette, I. Texier, Preparation and characterization of highly stable lipid nanoparticles with amorphous core of tuneable viscosity, J. Colloid Interface Sci. 360 (2011) 471-481, http://dx.doi.org/10.1016/j.jcis.2011.04.080.

[10] T. Courant, E. Bayon, H.L. Reynaud-dougier, C. Villiers, M. Menneteau, P.N. Marche, F.P. Navarro, Biomaterials Tailoring nanostructured lipid carriers for the delivery of protein antigens : physicochemical properties versus immunogenicity studies, Biomaterials 136 (2017) 29-42, http://dx.doi.org/10.1016/ j.biomaterials.2017.05.001.

[11] N. Anton, J.P. Benoit, P. Saulnier, Design and production of nanoparticles formulated from nano-emulsion templates-a review, J. Control. Release 128 (2008) 185-199, http://dx.doi.org/10.1016/j.jconrel.2008.02.007.

[12] I.J. Joye, D.J. McClements, Biopolymer-based delivery systems: challenges and opportunities, Curr. Trends Med. Chem. 16 (2016) 1026-1039.

[13] C. Tematio, M. Bassas-Galia, N. Fosso, V. Gaillard, M. Mathieu, M. Zinn, E. Staderini, S. Schintke, Design and characterization of conductive biopolymer nanocomposite electrodes for medical applications, Mater. Sci. Forum 879 (2017) 1921-1926, http://dx.doi.org/10.4028/www.scientific.net/MSF.879.1921.

[14] R. Shelma, C.P. Sharma, Development of lauroyl sulfated chitosan for enhancing hemocompatibility of chitosan, Colloids Surf. B Biointerfaces 84 (2011) 561-570, http://dx.doi.org/10.1016/j.colsurfb.2011.02.018.

[15] L. Mazzarino, L. Coche-Guérente, P. Labbé, E. Lemos-Senna, R. Borsali, On the mucoadhesive properties of chitosan-coated polycaprolactone nanoparticles loaded with curcumin using quartz crystal microbalance with dissipation monitoring, J. Biomed. Nanotechnol. 10 (2014) 787-794, http://dx.doi.org/10.1166/jbn.2014. 1768.

[16] R. Pandey, G.K. Khuller, Antitubercular inhaled therapy: opportunities, progress and challenges, J. Antimicrob. Chemother. 55 (2005) 430-435, http://dx.doi.org/ 10.1093/jac/dki027.

[17] A.L. Lainé, J. Gravier, M. Henry, L. Sancey, J. Béjaud, E. Pancani, M. Wiber, I. Texier, J.L. Coll, J.P. Benoit, C. Passirani, Conventional versus stealth lipid nanoparticles: formulation and in vivo fate prediction through FRET monitoring, J. Control. Release 188 (2014) 1-8, http://dx.doi.org/10.1016/j.jconrel.2014.05.042.

[18] F.P. Navarro, F. Mittler, M. Berger, V. Josserand, J. Gravier, F. Vinet, I. Texier, Cell tolerability and biodistribution in mice of indocyanine green-loaded lipid nanoparticles, J. Biomed. Nanotechnol. 8 (2012) 594-604, http://dx.doi.org/10.1166/ jbn.2012.1422.

[19] J.M. Rabanel, P. Hildgen, X. Banquy, Assessment of PEG on polymeric particles surface, a key step in drug carrier translation, J. Control. Release 185 (2014) 71-87, 
http://dx.doi.org/10.1016/j.jconrel.2014.04.017.

[20] P. Rivera-Gil, D. Jimenez De Aberasturi, V. Wulf, B. Pelaz, P. Del Pino, Y. Zhao, J.M. De La Fuente, I. Ruiz De Larramendi, T. Rojo, X.J. Liang, W.J. Parak, The challenge to relate the physicochemical properties of colloidal nanoparticles to their cytotoxicity, Acc. Chem. Res. 46 (2013) 743-749, http://dx.doi.org/10.1021/ ar300039j.

[21] S.T. Cole, R. Brosch, J. Parkhill, T. Garnier, C. Churcher, D. Harris, S.V. Gordon, K. Eiglmeier, S. Gas, C.E. Barry, F. Tekaia, K. Badcock, D. Basham, D. Brown, T. Chillingworth, R. Connor, R. Davies, K. Devlin, T. Feltwell, S. Gentles, N. Hamlin, S. Holroyd, T. Hornsby, K. Jagels, A. Krogh, J. McLean, S. Moule, L. Murphy, K. Oliver, J. Osborne, M.A. Quail, M.-A. Rajandream, J. Rogers, S. Rutter, K. Seeger, J. Skelton, R. Squares, S. Squares, J.E. Sulston, K. Taylor, S. Whitehead, B.G. Barrell, Deciphering the biology of Mycobacterium tuberculosis from the complete genome sequence, Nature 393 (1998) 537-544, http://dx.doi.org/10.1038/31159.

[22] A. Guillot, A.C. Couffin, X. Sejean, F. Navarro, M. Limberger, C.M. Lehr, Solid phase extraction as an innovative separation method for measuring free and entrapped drug in lipid nanoparticles, Pharm. Res. 32 (2015) 3999-4009, http://dx.doi.org/ 10.1007/s11095-015-1761-8.

[23] J.-C. Palomino, A. Martin, M. Camacho, H. Guerra, J. Swings, F. Portaels, Resazurin microtiter assay plate : simple and inexpensive method for detection of drug resistance in mycobacterium tuberculosis resazurin microtiter assay plate : simple and inexpensive method for detection of drug resistance in mycobacterium tuberculosis, Antimicrobail Agents Chemother. 46 (2002) 2720-2722, http://dx.doi.org/10. 1128/AAC.46.8.2720.

[24] E.K. Park, H.S. Jung, H.I. Yang, M.C. Yoo, C. Kim, K.S. Kim, Optimized THP-1 differentiation is required for the detection of responses to weak stimuli, Inflamm Res. 56 (2007) 45-50, http://dx.doi.org/10.1007/s00011-007-6115-5.

[25] G. Repetto, A. del Peso, J.L. Zurita, Neutral red uptake assay for the estimation of cell viability/cytotoxicity, Nat. Protoc. 3 (2008) 1125-1131, http://dx.doi.org/10. 1038/nprot.2008.75.

[26] M. Moros, S.G. Mitchell, V. Grazú, J.M. De la Fuente, The fate of nanocarriers as nanomedicines in vivo: important considerations and biological barriers to overcome, Curr. Med. Chem. 20 (2013) 2759-2778, http://dx.doi.org/10.2174/ 0929867311320220003.

[27] M. Goutayer, S. Dufort, V. Josserand, A. Royère, E. Heinrich, F. Vinet, J. Bibette, J.L. Coll, I. Texier, Tumor targeting of functionalized lipid nanoparticles: assessment by in vivo fluorescence imaging, Eur. J. Pharm. Biopharm. 75 (2010) 137-147, http://dx.doi.org/10.1016/j.ejpb.2010.02.007.

[28] G. Fotakis, J.A. Timbrell, In vitro cytotoxicity assays: comparison of LDH, neutral red, MTT and protein assay in hepatoma cell lines following exposure to cadmium chloride, Toxicol. Lett. 160 (2006) 171-177, http://dx.doi.org/10.1016/j.toxlet. 2005.07.001. 